\title{
Using the Guard-Stage-Milestone Notation for Monitoring BPMN-based Processes
}

\author{
Luciano Baresi, Giovanni Meroni, and Pierluigi Plebani \\ Politecnico di Milano - Dipartimento di Elettronica, Informazione e Bioingegneria \\ Piazza Leonardo da Vinci, 32 - 20133 Milano, Italy \\ [firstname]. [lastname] @polimi.it,
}

\begin{abstract}
Business processes are usually designed by means of imperative languages to model the acceptable execution of the activities performed within a system or an organization. At the same time, declarative languages are better suited to check the conformance of the states and transitions of the modeled process with respect to its actual execution. To avoid defining models twice from scratch to cope with both the process enactment and its monitoring, this paper proposes an approach for translating BPMN process models to E-GSM ones: an extension of the Guard-Stage-Milestone artifact-centric notation. The paper also shows how a monitoring engine based on E-GSM specifications can detect anomalies during the execution of the process and classify them according to different levels of severity, that is, with respect to the impact on the outcome of the process.
\end{abstract}

Keywords: Guard-Stage-Milestone, Artifact-centric processes, BPMN, Process execution monitoring

\section{Introduction}

Process modeling represents one of the most crucial activities in Business Process Management and the goal of the resulting model is twofold. On the one hand, a business process model describes a portion of the world as it is (or as we want it to be) using a formalism easy to understand by all the relevant stakeholders (e.g., process owners and process users). On the other hand, a business process model - if properly defined in all of its parts - feeds the engine that will enact its execution. To this aim, imperative control-flow based languages are widely adopted, as their constructs and the underlying semantics are very intuitive. Among them, BPMN nowadays represents one of the most used notation adopted by both business and technical people.

However, when used for monitoring the execution of a process at run-time, imperative languages manifest a significant limitation: when a violation in the control flow occurs, an imperative process engine treats such a violation as an unhandled exception and stops monitoring the process until a user manually fixes the issue. This is not always desirable, especially when the engine has no control on the monitored process, which would continue its execution even though the 
engine stopped. Declarative languages, on the other hand, do not have the notion of strict control flow. Therefore, declarative engines can both report deviations in the control flow and continue monitoring the process.

The goal of this paper is to mediate between these two perspectives by proposing a solution to monitor the execution of distributed control-flow processes modeled in BPMN, that relies on a monitoring system based on the artifactcentric Guard-Stage-Milestone (GSM) declarative language 6]. In particular, we start from a BPMN process, which is easy to conceive, and we transform it into a model defined using E-GSM, our extension of GSM. This transformation preserves control flow information, but such information, which is prescriptive in BPMN, becomes descriptive. Deviations from the "original" execution flow can easily be detected at run-time during the process enactment by analyzing the artifacts, that contain information about how the process is evolving, and represent the states through which the process should evolve during execution.

The adoption of E-GSM to drive the process monitoring introduces the following advantages. E-GSM allows one to define conditions both on the process and on external data to trigger the execution and termination of activities. Therefore, the monitoring platform can infer when activities are executed based on information coming from the environment, thus being not limited to explicit messages. Furthermore, E-GSM allows one to identify the results of the execution of the activities within the process model, and consequently it permits the identification of the activities that are incorrectly executed, if any.

The rest of the paper is structured as follows. Section 2 discusses how we extended GSM into E-GSM to enable a data-artifact driven process monitoring solution. Section 3 introduces the set of rules we defined to translate BPMN elements into equivalent E-GSM ones. Section 4 validates our work by showing how to apply the approach on a real business process in the domain of logistics. Section 5 surveys the state of the art, and Section 6 concludes the paper.

\section{E-GSM}

The GSM notation is a declarative language that allows one to model artifactcentric processes by defining conditions that determine the activation and termination of activities, called Stages. With respect to other declarative languages, like Declare [14, such conditions are not limited to dependencies among activities. Instead, they are based on events, which can be external (e.g., sent or received messages), or internal (e.g., termination of activities), to the process. Starting from the standard GSM notation and our preliminary work 2, we propose E-GSM, an extension to GSM where we distinguish between Data Flow Guards and Process Flow Guards and we add Fault Loggers.

The goal of this extension is to include information on the normal flow, that is, the expected behavior of the process, or happy path, in the artifact-centric process model. To this aim, the process model includes the dependencies among activities in terms of control flow. Being a declarative language, E-GSM does not use control flow information to enforce a specific execution path among activities. 


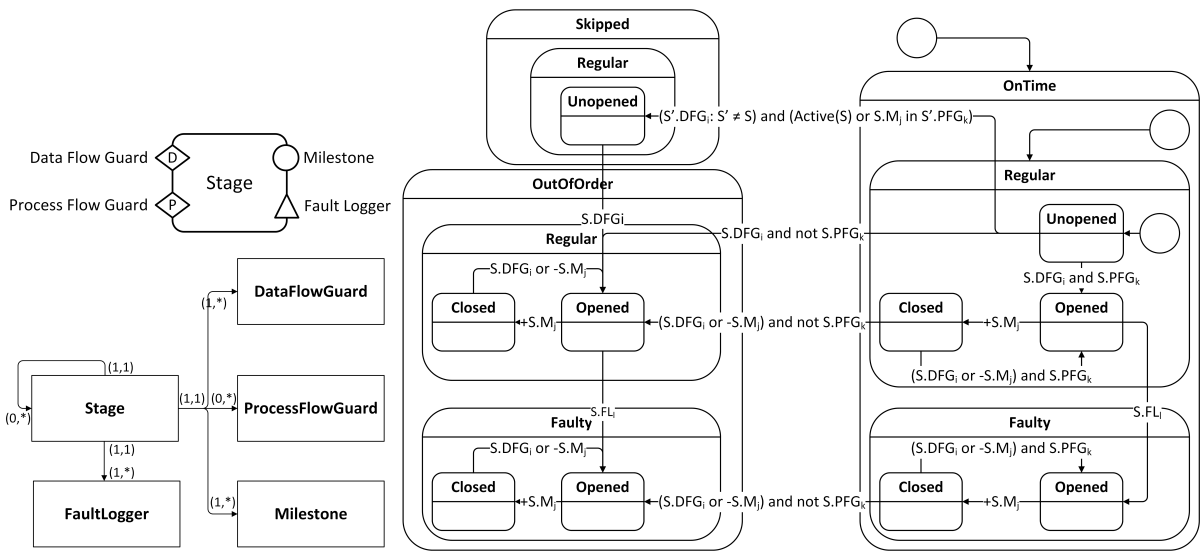

Fig. 1. E-GSM meta-model (bottom left), graphical representation (top left) and lifecycle of a Stage (right).

Instead, it uses such information to let the process engine detect deviations between the happy path and how the process is actually executed.

The left portion of Fig. [1] shows a simplified version of the meta-model behind E-GSM, along with the graphical representation of its main elements. The original definition of GSM comprises Stages, Guards, and Milestones. A Stage represents the unit of work that can be executed in a process instance. A Stage can have one or more nested Stages, or it can be atomic, thus representing a single task. A Stage may be decorated with one or more Guards and Milestones.

A Guard (Data Flow Guard in E-GSM) is an Event-Condition-Action (ECA) rule ${ }^{1}$ If true, the associated Stage is declared opened. A Milestone is another ECA rule. If true, the Stage is declared closed. A Milestone may also have an invalidator: a boolean expression that can invalidate the Milestone and reopen the Stage.

In the proposed extension, a Stage can now also be decorated with Process Flow Guards and Fault Loggers. A Process Flow Guard is a boolean expression that predicates on the activation of the Data Flow Guards and Milestones used to map the expected control flow. The expression is evaluated once one of the Data Flow Guards of the associated Stage is triggered, and before the Stage becomes opened. If the expression is true, the Stage complies with the expected execution, otherwise the Stage has been activated without respecting the normal flow.

A Fault Logger is an ECA rule. If true, the associated Stage is declared faulty because something went wrong during the execution of the activity. A

\footnotetext{
${ }^{1}$ An ECA rule is an [on e] [if c] expression, that is triggered when an event $e$ occurs and the condition $c$ is true. When [on $e$ ] is missing, the ECA is triggered once $c$ becomes true, when [if $c$ ] is missing, the ECA is triggered once $e$ occurs.
} 
faulty Stage does not imply its termination, as the termination is only determined by Milestones.

The right portion of Fig. 1 sketches the lifecycle of an E-GSM Stage organized around three main orthogonal execution perspectives: outcome, compliance, and status ${ }^{2}$

The Execution outcome captures the situation of a Stage, which can be either regular (none of its Fault Loggers has ever been triggered) or faulty (at least one of its Fault Loggers has been triggered, A. FL $L_{1}$ ).

The Execution compliance captures the compliance of each Stage with the normal flow. A Stage is declared onTime by default. It can become outOfOrder (according to the normal flow) when one of its Data Flow Guards is triggered but none of its Process Flow Guards holds (A.DFG and not (A.PFG)). If a Stage $S^{\prime}$ ' is declared outOfOrder, every other on Time Stage S that would trigger one of the Process Flow Guards of $S^{\prime}\left(S . M_{j}\right.$ or Active $\left.(S) \in S^{\prime} \cdot P_{F} G_{k}\right)$ is declared skipped. If a Stage is skipped, once one of its Data Flow Guards is triggered $\left(\mathrm{S} \cdot \mathrm{DFG}_{\mathrm{i}}\right)$, it becomes outOfOrder.

The Execution status captures the status of a Stage: unopened, opened or closed. A Stage is unopened if its Data Flow Guards have never been triggered. A Stage can become opened only if it is unopened or closed and the parent Stage is opened. In addition, at least one of its Data Flow Guards must be triggered $\left(\mathrm{S} . \mathrm{DFG}_{\mathrm{i}}\right)$. A Stage becomes closed if it is opened and a Milestone is achieved $\left(+\mathrm{S} . \mathrm{M}_{\mathrm{j}}\right)$, or if the parent Stage becomes closed.

The combination of these three perspectives says that the whole lifecycle assumes that a Stage is initially onTime, regular, and unopened. Data Flow Guards drive the change of execution status. Fault Loggers drive the outcome, while Process Flow Guards are in charge of the compliance. With respect to Standard GSM, E-GSM interprets reopening a closed Stage as a new iteration of that process portion. Therefore, once a parent Stage is reopened (i.e., it moves from closed to opened), the lifecycle of all its child Stages will restart from scratch.

Thank to these three perspectives, it is possible to detect at runtime when a deviation in the execution of a process occurs and which stages are involved. This enables a classification that predicates on the lifecycle of all stages to evaluate how severely variations during execution affect the outcome of the process. For example, Table 1 reports a possible classification of severity that can be modified according to any specific scenario: None, if all activities are executed at the right time and their execution was successful. Low, if the process terminated, the expected control flow was not respected, yet no activity was skipped and they were all successfully executed. Medium-low, if an activity was incorrectly executed, but the expected control flow was respected. Medium, if the process

\footnotetext{
${ }^{2}$ In this paper we use the the notation introduced in [6], so we write $\mathrm{S} . \mathrm{DFG}_{\mathrm{i}}, \mathrm{S} . \mathrm{PFG}_{\mathrm{k}}$, S.FL 1 to indicate the activation of a Data Flow Guard, Process Flow Guard, or a Fault Logger associated with Stage $S,+S . M_{j}\left(-S . M_{j}\right)$ to indicate the achievement (invalidation) of a Milestone $M_{j}, S . M_{j}$ to indicate that Stage $S$ is closed and a Milestone $M_{j}$ is achieved, and Active( $S$ ) to indicate that Stage $S$ is opened.
} 
Table 1. Severity levels. $S_{x} . o, S_{x} . c$ and $S_{x} . s$ indicate the state of stage $S_{x}$, along with the execution outcome, compliance and status respectively.

\begin{tabular}{|c|c|c|c|}
\hline Severity & $\begin{array}{c}\text { Execution } \\
\text { outcome }\left(S_{y} . o\right)\end{array}$ & $\begin{array}{c}\text { Execution } \\
\text { compliance }\left(S_{z} \cdot c\right)\end{array}$ & $\begin{array}{c}\text { Execution } \\
\text { status }\left(S_{x} . s\right)\end{array}$ \\
\hline None & $\forall S_{y}: S_{y} \cdot o=$ regular & $\forall S_{z}: S_{z} \cdot c=$ onTime & $\begin{array}{c}\forall S_{x}: S_{x} . s=\text { unopened } \\
\vee S_{x} . s=\text { opened } \\
\vee S_{x} . s=\text { closed }\end{array}$ \\
\hline Low & $\forall S_{y}: S_{y} \cdot o=$ regular & $\exists S_{z}: S_{z} \cdot c=$ outOfOrder & $\begin{array}{c}\forall S_{x}: S_{x} . s=\text { unopened } \\
\quad \vee S_{x} . s=\text { closed }\end{array}$ \\
\hline Medium-low & $\exists S_{y}: S_{y} \cdot o=$ faulty & $\forall S_{z}: S_{z} \cdot c=$ onTime & $\begin{array}{c}\forall S_{x}: S_{x} . s=\text { unopened } \\
\vee S_{x} . s=\text { opened } \\
\vee S_{x} . s=\text { closed }\end{array}$ \\
\hline Medium & $\forall S_{y}: S_{y} \cdot o=$ regular & $\begin{array}{c}\exists S_{z}: S_{z} \cdot c=\text { outOfOrder } \\
\vee S_{z} \cdot c=\text { skipped }\end{array}$ & $\exists S_{x}: S_{x} . s=$ opened \\
\hline Medium-high & $\forall S_{y}: S_{y} \cdot o=$ regular & $\exists S_{z}: S_{z} \cdot c=$ skipped & $\begin{array}{c}\forall S_{x}: S_{x} . s=\text { unopened } \\
\quad \vee S_{x} . s=\text { closed }\end{array}$ \\
\hline High & $\exists S_{y}: S_{y} \cdot o=$ faulty & $\begin{array}{c}\exists S_{z}: S_{z} \cdot c=\text { outOfOrder } \\
\vee S_{z} \cdot c=\text { skipped }\end{array}$ & $\begin{array}{c}\forall S_{x}: S_{x} . s=\text { unopened } \\
\vee S_{x} . s=\text { opened } \\
\vee S_{x} . s=\text { closed }\end{array}$ \\
\hline
\end{tabular}

is still in progress and, during execution, the expected control flow was not respected. Medium-high, if the process terminated and an activity was skipped. $H i g h$, if an activity was incorrectly executed and no corrective action was taken (i.e., at least another activity was either skipped or incorrectly executed).

This classification assumes that all stages have the same importance. However, weights can be introduced to differentiate the influence of each specific stage on the process, or metrics taken from the conformance checking domain [18] can be adopted.

\section{Transformation Rules}

The aforementioned semantics of E-GSM is then used in 13 transformation rules [1] to translate a BPMN process model into an E-GSM one.

These transformation rules are applicable to every BPMN process model that complies with a workflow net [1], that is, the process has only one start event and only one end event, and it always terminates (soundness). It is worth noting that the control flow is always captured by Process Flow Guards, and as such it is never enforced. This allows the E-GSM model to continue monitoring a process even if violations in the control flow occur.

\subsection{Basic Elements}

The transformation rules defined for basic elements are presented in Fig. 2

Rule 1 A BPMN Activity A is translated into a Stage A with one or more Data Flow Guards $\left(A . D F G_{i}\right)$ and one or more Milestones $\left(A . M_{j}\right)$. 


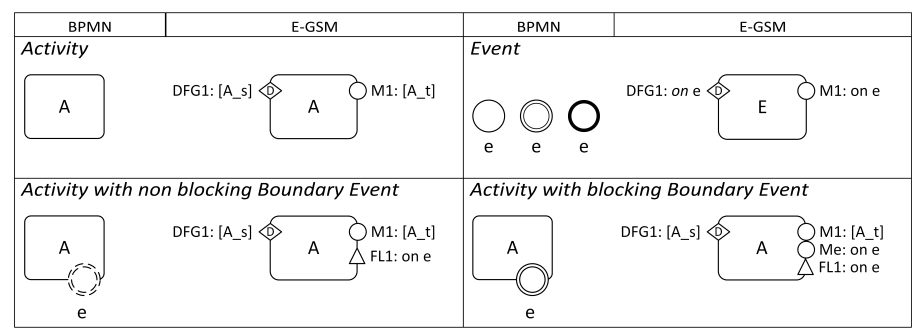

Fig. 2. BPMN to E-GSM transformation rules for basic elements.

Producing the conditions associated with those Data Flow Guards and Milestones is far from trivial 3. They depend on the associated data objects and, if the activity is a task, on its type (i.e., receive or user task). In case of a generic task, placeholders A_s and A_t are associated with, respectively, A.DFG1 and A.M1 to represent the explicit start and termination of the activity. If the activity is a sub-process, A.DFG $\mathrm{i}_{i}$ and $\mathrm{A} . \mathrm{M}_{j}$ are then derived from the structure of the sub-process and from its elements, as explained in the following.

Rule 2 A BPMN Start, End or Intermediate Event e is translated into a Stage $E$ where E.DFG1 and E.M1 have the occurrence of the event as condition.

Rule 3 A BPMN Activity A with a non-interrupting Boundary Event e attached is translated into a Stage A according to Rule 1 with A.FL1 having the occurrence of the event as condition (i.e., on e).

Rule 4 A BPMN Activity A with an interrupting Boundary Event e attached is translated into a Stage A according to Rule 1 with an additional Milestone A. Me and A.FL1 having the occurrence of the event as condition.

\subsection{Normal Flow}

The combination of the above rules for basic elements allows one to translate well-structured business process models [17. In particular, we focus on five types of blocks, defined starting from the classical control flow patterns [19]:

- A sequence block is made of linked activities, events and other blocks without splits or merges. It corresponds to pattern sequence.

- A parallel block organizes activities, events, and other blocks in two or more parallel threads resulting from the combination of patterns parallel split and synchronization.

- A conditional exclusive block organizes activities, events, and other blocks in two or more branches resulting from a combination of patterns exclusive choice and simple merge.

- A conditional inclusive block organizes activities, events, and other blocks in two or more branches resulting from a combination of patterns multi-choice and structured synchronized merge. 
- A loop block organizes activities, events, and other blocks according to pattern structured loop.

For each of these blocks, we delivered proper transformation rules in [11. A graphical representation of them is reported in Fig. 3. Due to space constraints, in this paper we will only describe in detail how sequence, conditional exclusive, and loop blocks are translated.

Rule 5 A sequence block corresponds to a Stage Seq that includes $S_{x}$ inner Stages obtained by applying the transformation rules to all the elements (i.e., Activities, Events, inner blocks) that belong to the block.

- In addition to the existing Process Flow Guards, each inner stage has $S_{x} . P F G 1$ to state that none of its Milestones is achieved, and at least one of the Milestones of the element that directly precedes it (if present) is achieved.

- Seq has a set Seq.DFG that includes all $S_{x} . D F G_{i}$, and a Milestone Seq.M1 that requires that, for all $S_{x}$, at least one $S_{x} . M_{j}$ be achieved.

Rule 6 A conditional exclusive block is translated into a Stage Exc that includes all the Stages obtained by applying Rule 5 to all its branches, which result in $S_{x}$ inner Stages.

- For each $S_{x}, S_{x} . P F G 1$ is added to check that no $S_{x} . M_{j}$ has already been achieved, that the condition on the branch from which $S_{x}$ is produced (if present) is satisfied, and that none of the other inner Stages is opened (i.e., not Active ( $\left.S_{y}\right)$ where $\left.y \neq x\right)$.

- Exc has a set Exc.DFG that includes all $S_{x} . D F G_{i}$, and a Milestone Exc.M1 that requires that, for at least one $S_{x}$, one $S_{x} . M_{j}$ be achieved, and the condition on the branch from which $S_{x}$ is produced (if present) be satisfied, as long as none of the other inner Stages is opened.

Rule 7 A loop block is translated into two Stages, Ite and Loop. Ite includes $S_{x}$ inner Stages obtained by applying Rule 5 to all the branches within the loop block. One of these stages is a forward Stage, that is, its control flow goes in the same direction as the control flow that includes the loop block. The others are backward Stages.

- For all the inner Stages, $S_{x}$.PFG1 is added to check that no $S_{x} . M_{j}$ is already achieved. Moreover, if $S_{x}$ is a backward stage, $S_{x}$.PFG1 also requires that the condition on the branch (if present) be satisfied, and that one of the Milestones of the forward stage be achieved.

- Ite has a set Ite.DFG that includes all $S_{x} . D F G_{i}$, and two Milestones, where:

- Ite.M1 requires that one of the Milestones of the forward Stage be achieved and the exit condition of the loop (if present) be satisfied, as long as no backward Stage is opened.

- Ite.M2 requires that one of the Milestones of the forward Stage be achieved and, for at least a backward Stage, one of its Milestones be achieved and the condition on that branch (if present) be satisfied, as long as none of the other backward Stages is opened. 


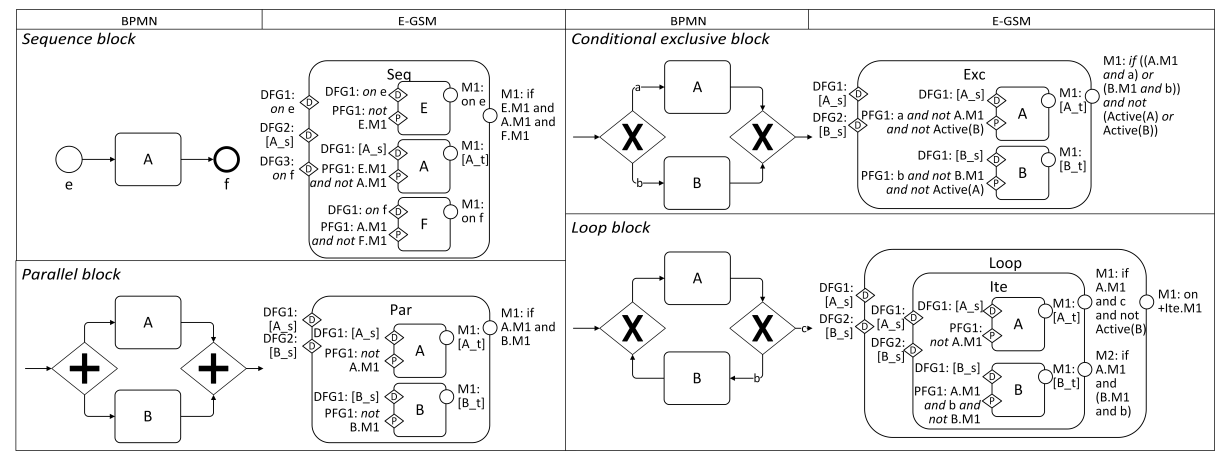

Fig. 3. BPMN to E-GSM transformation rules for normal flow blocks (due to space constraints, the conditional inclusive block is not presented).

Stage Loop includes Ite and has Loop.DFG $=$ Ite. $D F G$ and Loop. $M=$ on Ite.M1 (i.e., the process can exit the loop).

The iteration Stage Ite has no Process Flow Guards since it is supposed to be executed multiple times and, every time it becomes opened, a new iteration of the loop is carried out. Thus, Ite is opened when at least one of its inner Stages can be opened too, and it is closed when either the process can exit the loop (Ite.M1 is achieved), or when an iteration is complete (Ite.M2 is achieved).

\subsection{Exceptional Flow}

BPMN supports the management of foreseen exceptions through boundary events, that is, events directly attached to activities. These events, like split gateways, determine a branching of the control flow into an exceptional flow, which leaves the boundary event, and a normal flow, to continue the execution from the activity. If the foreseen exception occurs while executing the activity, the attached boundary event activates the exceptional flow. A dedicated set of rules shown in Fig. 4 is thus required to preserve this behavior in E-GSM models. Again, we refer to [11] for the details.

Interrupting boundary events cause the normal and exceptional flows to be mutually exclusive, therefore we expect them to be merged by an exclusive merge gateway at the end. This requires that two additional blocks, called forward exception handling and backward exception handling, respectively, be defined. The forward exception handling block comprises an interrupting boundary event, and a simple merge, defined with a BPMN exclusive gateway, that merges the exceptional control flow and the portion of the normal control flow that follows the activity to which the boundary event is attached. Its behavior is similar to the one of the conditional exclusive block, with the exception of the branch condition, which predicates on the achievement of the milestone derived from the boundary event. The backward exception handling block comprises an interrupting boundary event and a simple merge, defined with a BPMN exclusive 


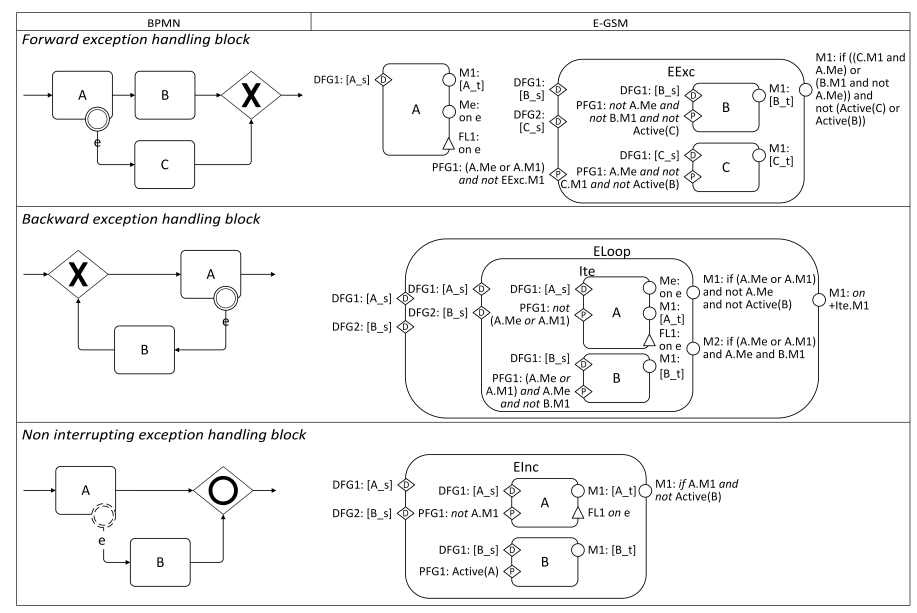

Fig. 4. BPMN to E-GSM transformation rules for handling exceptions.

gateway, that merges the exceptional control flow and the portion of the normal control flow that precedes the activity to which the boundary event is attached. This block produces a loop that allows one to re-execute part of the normal control flow if the boundary event is triggered, and therefore it is translated similarly to a loop block.

In BPMN, boundary events could also be non interrupting, that is, they activate the exceptional control flow without terminating the associated activity. Therefore, the elements within the exceptional control flow can run in parallel with the normal flow that starts from the activity the boundary event is associated with. Since we expect these potentially simultaneous control flows be merged by an inclusive merge gateway, the transformation requires an additional block, called non interrupting exception handling block. This new block comprises a non interrupting boundary event to split the execution flow into an exceptional flow and the continuation of the normal one, and a structured synchronized merge, defined with a BPMN inclusive gateway, to merge the two flows in case the exception occurred.

\section{Validation}

The transformation rules introduced in the previous section allow any well-structured BPMN process model to be translated into E-GSM. To prove it, we developed a BPMN to E-GSM prototype translator 3 , where the transformation rules are implemented in ATL (ATLAS Transformation Language [7]), and validated - and refined - the proposed rules against several BPMN business processes with different levels of complexity. A formal verification about the equivalence between BPMN processes and their correspondent E-GSM is under study and

${ }^{3}$ The tool is publicly available at https://bitbucket.org/polimiisgroup/ bpmn2egsm 


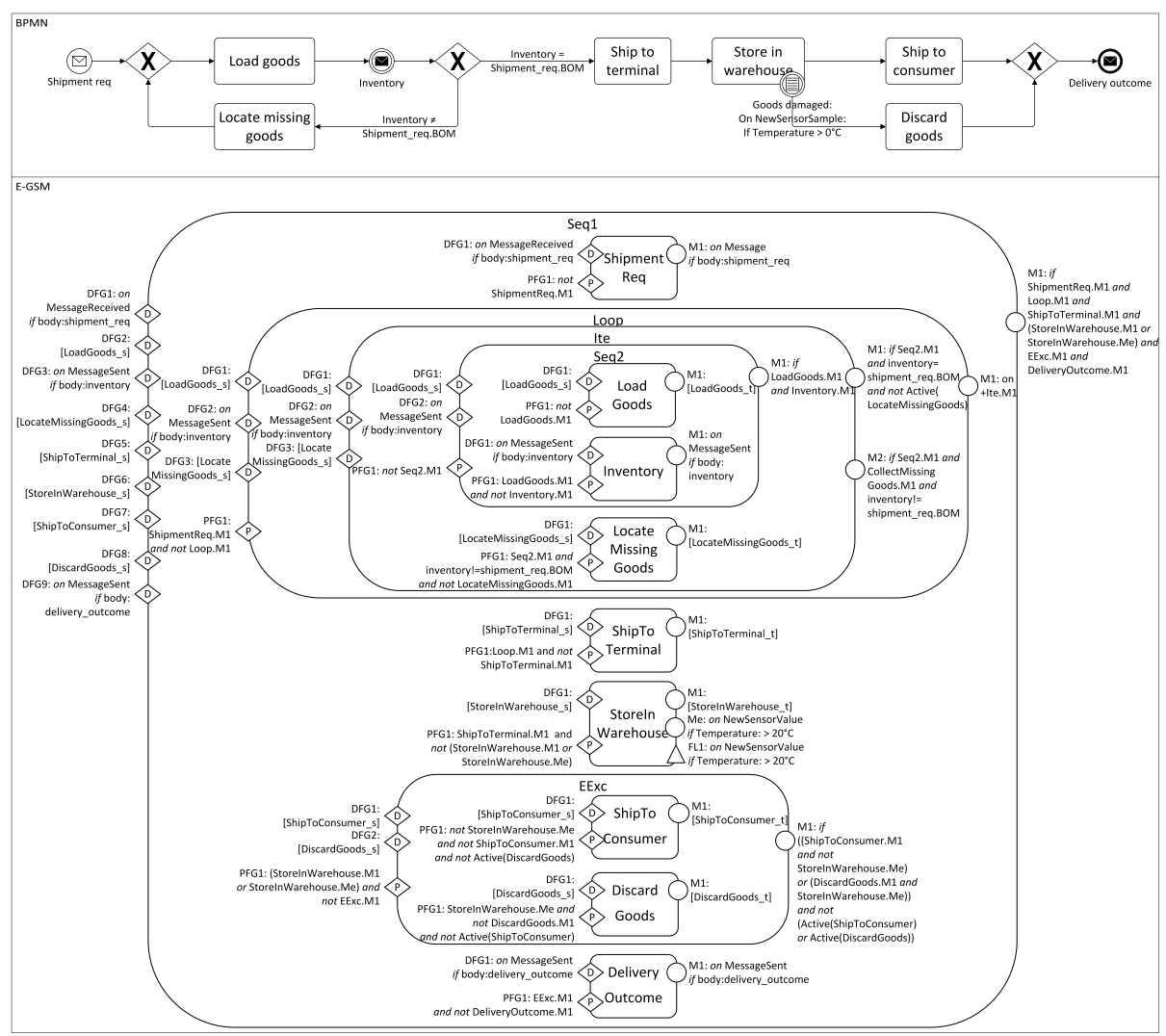

Fig. 5. BPMN and E-GSM models of the example shipping process.

it aims to check if all the traces that a BPMN process can produce are also considered as satisfied in the E-GSM model.

Among these test processes, here we concentrate on an example taken from the logistics domain, which is shown at the top of Fig. 5, to better explain the advantages of adopting E-GSM to monitor the execution of complex (distributed) processes. A pharmaceutical company $M$ has to ship drugs (that are highly susceptible to temperature variations) to one of its customers $N$. To do so, it relies on two shipping companies $R$ and $T$ for, respectively, rail and truck transportation, and on an inland terminal $I$ for changing means. The shipping process starts when a shipment request by $N$ is received, and comprises four main phases: (i) loading goods into a thermally-insulated shipping container; (ii) shipping such a container to $I$ by rail; (iii) temporarily storing the container in a temperaturecontrolled warehouse; (iv) delivering the goods to the customer's site by truck. Before starting phase (ii), an inventory report of the contents of the container must be produced, and it must be compared with the bill of materials included with the shipment request. If some products are missing, they must be located and loaded onto the container, and a new report must be produced. Furthermore, if the goods are exposed to a temperature higher than $20^{\circ} \mathrm{C}$ during phase (iii), 
they must be discarded and the whole process must be aborted. Our translator produces the E-GSM model shown at the bottom of Fig. 5 .

Since all these activities interact with the shipping container, we can think of it as the process coordinator (i.e., the element that interacts with all the parties and has complete visibility on the whole process). To make the container process-aware, we can exploit the Internet of Things paradigm by equipping it with a single board computing device, sensors and a network interface, thus transforming it into a smart object (i.e., smart container). However, being the container completely passive, it cannot enforce the execution flow modeled in the process, and it needs information to identify when each activity is being executed. For this reason, a traditional process engine would be unsuited to monitor this process. On the other hand, an E-GSM engine 4 running onto the smart container would solve this problem: By predicating on on-board sensor values or explicit messages, Data Flow Guards, Process Flow Guards, Milestones and Fault Loggers can be triggered, and the execution of the process be monitored. This way, once a violation in the execution occurs, the E-GSM engine can report to stakeholders which activities are affected, and how severely the whole process is affected by such an incident.

To show how process monitoring can take advantage of the E-GSM model, we describe three possible scenarios.

\subsection{An Error-Free Execution}

Once the shipment request is received, Seq1.DFG1 is triggered and, consequently, Seq1 becomes opened (thus starting the process). This first triggers ShipmentReq.PFG1, then ShipmentReq. DFG1, which causes ShipmentReq to become opened, and finally ShipmentReq.M1 be achieved, which moves ShipmentReq to the closed state, and triggers Loop.PFG1.

When $R$ loads the goods onto the container, a notification is sent to the engine, which triggers Loop.DFG1, then Ite.DFG1, Seq2.PFG1, Seq2.DFG1, LoadGoods.PFG1, and finally LoadGoods.DFG1, which moves Loop, Ite, Seq2 and LoadGoods to the opened state. After finishing loading the goods, the operator sends another notification, thus making LoadGoods.M1 be achieved, which triggers Inventory.PFG1 and moves LoadGoods to the closed state. It then produces the inventory of the loaded goods, which triggers Inventory.DFG1, and then makes Inventory.M1 be achieved, which makes Seq2.M1 achieved too, causing Inventory and Seq2 to move to the closed state. Being the inventory consistent with the bill of materials included in the shipment request, also Ite.M1 and, consequently, Loop.M1, are achieved, moving Ite and Loop to the closed state, and triggering ShipToTerminal.PFG1.

Once the rail shipping begins, $R$ sends a notification, which triggers ShipToTerminal.DFG1 and moves ShipToTerminal to the opened state. When the container is delivered to $I$, another notification is sent, which makes ShipToTerminal. M1 become achieved, moving ShipToTerminal to the closed state and triggering

\footnotetext{
${ }^{4}$ A prototype E-GSM engine is currently under development
} 
StoreInWarehouse.PFG1. Similarly $I$ sends a notification when the container is put in the warehouse and when $T$ is ready to pick it up, thus triggering StoreInWarehouse.DFG1, achieving StoreInWarehouse.M1, triggering EExc.PFG1, and moving StoreInWarehouse to the opened state at first, and then to the closed state. After hooking the container to its truck, a notification is sent by $T$. That notification triggers EExc.DFG1, then ShipToCustomer.PFG1, and finally ShipToCustomer.DFG1, thus moving stages EExc and ShipToCustomer to the opened state. Once $T$ delivers the goods to $N$, another notification is sent. That notification causes the achievement of ShipToCustomer.M1, which makes EExc.M1 become achieved too, thus moving ShipToCustomer and EExc to the closed state and triggering DeliveryOutcome.PFG1.

Finally, once the goods are inspected by $N$, a report of the shipment is produced, which triggers DeliveryOutcome.DFG1, moving DeliveryOutcome to the opened state, and then causes the achievement of DeliveryOutcome.M1, which causes the achievement of Seq1.M1 too, thus moving DeliveryOutcome and Seq1 to the closed state and, since Seq1 represents the whole process, terminating the monitoring activity. Once the process concludes, $N$ queries the smart container and finds out that the severity level of the process is None, since all stages are in state either unopened or closed, their compliance is on Time, and their outcome is regular. Therefore, $N$ accepts the goods.

\subsection{A Catastrophic Execution}

A second example shows how the system can monitor an incorrect execution of the process. During phase (iii), the warehouse cooling system breaks down, and the temperature of the goods goes beyond $20^{\circ} \mathrm{C}$. Being the container equipped with a temperature sensor, the E-GSM engine is able to detect such an event and consequently triggers both StoreInWarehouse.FL1 and StoreInWarehouse.Me, which move StoreInWarehouse to the faulty and closed states. This changes the severity level of the process from none to medium-low, since a faulty stage exists, but all stages are still onTime. Being StoreInWarehouse closed and StoreInWarehouse. Me achieved, DiscardGoods.PFG1 is also triggered.

Instead of discarding the goods, $I$ ignores that accident, and delivers the goods to $N$. This moves ShipToConsumer to state outOfOrder, since ShipToConsumer.DFG1 is triggered before ShipToConsumer.PFG1 becomes active. This causes the severity level of the process to become high, since there are both a faulty stage (StoreInWarehouse), and an outOfOrder one (ShipToConsumer).

Once $N$ receives the goods, it queries the smart container and, since the severity level of the process is high, decides to immediately inspect its content, thus discovering that the goods have been spoiled. Therefore, it sends them back to $M$. In turn, $M$ identifies that StoreInWarehouse is in the faulty state, and that ShipToConsumer is outOfOrder. Thank to this information, $M$ is able to charge $I$ a penalty for having spoiled the goods and not having reported that accident. Note that had $T$ queried the smart container, it would have seen that the severity level was medium-low, since StoreInWarehouse was in faulty state, and could have avoided delivering the container to $N$. 


\subsection{A Troublesome yet Recoverable Execution}

Let us now focus on a less critically incorrect execution of the process. In this case, the inventory of the container is not consistent with the bill of materials, which causes LocateMissingGoods.PFG1 to be triggered. However, $R$ does not check the inventory and immediately begins shipping the container to $I$, which moves ShipToTerminal to the outOfOrder state, since ShipToTerminal.DFG1 is triggered before ShipToTerminal.PFG1 becomes active. This changes the severity level of the process from none to medium, as there are both opened stages (Seq1, Loop and Ite) and an outOfOrder one (ShipToTerminal).

Once $N$ receives the goods, it queries the smart container and finds out that the severity level is still medium (since the missing goods were not collected and loaded onto the container, stages Seq1, Loop and Ite are still opened). So, it inspects the contents, discovers that some of them are missing, and asks $M$ to ship the missing ones for free. By querying the smart container, $M$ finds out that, even though the inventory did not match the shipping request, missing goods were never collected and loaded onto the smart container (i.e., LocateMissingGoods has not been executed even though LocateMissingGoods.PFG1 was satisfied), and the shipment continued anyway (i.e., ShipToTerminal is in state outOfOrder). Because of this information, $M$ can blame $R$ for having shipped the goods without checking the inventory first. Note that the severity level (medium) reflects the results of the process: being at least part of the goods successfully delivered, $M$ did not experience a complete loss as in the previous case, where all the goods were spoiled, and the truck shipment was done pointlessly.

\section{Related Work}

Köpke et al. 8 propose transformation rules that transform a BPMN process model into a GSM equivalent. While we have borrowed from these rules the idea of transforming blocks into nested Stages, our transformation rules produce completely different expressions for Guards and Milestones. The reason behind such a discrepancy is that we are interested in identifying control flow violations, and not in forcing the process to rigidly follow a given execution flow, which is what is pursued in [8. Eshuis et al. 4] define a semi-automated approach that starts from UML Activity Diagrams and produces a data-centric process model in GSM. They capture the lifecycle of the data artifacts referred to in the UML process model, and exploit control flow information to render it in GSM. Similarly, Kumaran et al. [9] and Meyer et al. [13] propose a language-agnostic algorithm to derive the lifecycle of artifacts based on an imperative process model. This is possible as long as each activity has input and output information entities explicitly defined in the model. Our work differentiates from [4, 9], and 13, which use control flow information to model the interactions among data artifacts, by keeping such information in the target process model to assess compliance. Popova et al. [16] define a translator from Petri Nets to GSM. The main purpose of that translator is to transform the outcome of process mining algorithms, which is often represented as a Petri Net, to a GSM model. This 
way, process mining techniques can be used to identify business artifacts that the translator represents in a language that is easier to understand by domain experts than Petri Nets.

Concerning the integration of both activity and data-centric perspectives in business processes, Künzle et al. [10] propose a framework that maps portions of data structures to activities and use control flow information to define how such data objects should be manipulated. Similarly, Meyer et al. 12 propose a methodology to model both the control flow and data dependencies by extending BPMN data artifacts to define dependecines among all data items manipulated in a process. Both [10] and [12] use control flow information in a prescriptive way, while E-GSM uses it in a descriptive way to detect deviations from the original definitions during execution.

Conformance checking is the discipline that aims at identifying inconsistencies among a process model and its execution 18. To do so, the process model is checked against high level execution logs, which report when and if activities have been executed. Our solution differs from this approach as it is able to autonomously identify when activities start or end, without relying on an execution log. Furthermore, it is able to detect deviations at runtime, whereas most process compliance techniques are applicable only when the process terminates.

\section{Conclusions and Future Work}

This paper extends the Guard-Stage-Milestone (GSM) notation to embed control flow information in the process model definition, presents a solution for transforming BPMN models into equivalent E-GSM ones, and shows how the derived E-GSM process model can be used to identify when activities are executed, to keep track of violations in the execution flow, and to evaluate the overall execution of a process along with different levels of severity.

As for our future work, we will investigate how to improve Rule 1 and Rule 2 by taking into account the nature of activities (i.e., receive tasks or user tasks) and events (i.e., timer, signal, etc.), and their associated data objects. We will also propose additional transformation rules to derive the E-GSM Information Model, which is not considered in this work, from data objects and implicit information defined in BPMN process models, which may also influence the definition of severity levels. In parallel, we will continue applying the proposed solution and assessing it on real industrial examples.

\section{Acknowledgments}

This work has been partially funded by the Italian Project ITS Italy 2020 under the Technological National Clusters program.

\section{References}

1. Van der Aalst, W.M.: Verification of workflow nets. In: Application and Theory of Petri Nets 1997, pp. 407-426. Springer (1997) 
2. Baresi, L., Meroni, G., Plebani, P.: A gsm-based approach for monitoring crossorganization business processes using smart objects (2015), accepted for publication

3. Cabanillas, C., Baumgrass, A., Mendling, J., Rogetzer, P., Bellovoda, B.: Towards the enhancement of business process monitoring for complex logistics chains. In: Business Process Management Workshops. pp. 305-317. Springer (2014)

4. Eshuis, R., Van Gorp, P.: Synthesizing data-centric models from business process models. Computing pp. 1-29 (2015)

5. Fahland, D., Lübke, D., Mendling, J., Reijers, H., Weber, B., Weidlich, M., Zugal, S.: Declarative versus Imperative Process Modeling Languages: The Issue of Understandability. In: Enterprise, Business-Process and Information Systems Modeling. pp. 353-366. Springer (2009)

6. Hull, R., Damaggio, E., Fournier, F., Gupta, M., Heath, Fenno(Terry), I., Hobson, S., Linehan, M., Maradugu, S., Nigam, A., Sukaviriya, P., Vaculin, R.: Introducing the guard-stage-milestone approach for specifying business entity lifecycles. In: Web Services and Formal Methods, Lecture Notes in Computer Science, vol. 6551, pp. 1-24. Springer (2011)

7. Jouault, F., Allilaire, F., Bézivin, J., Kurtev, I.: Atl: A model transformation tool. Science of computer programming 72(1), 31-39 (2008)

8. Köpke, J., Su, J.: Towards ontology guided translation of activity-centric processes to gsm (2015), accepted for publication

9. Kumaran, S., Liu, R., Wu, F.Y.: On the duality of information-centric and activitycentric models of business processes. In: Advanced Information Systems Engineering. pp. 32-47. Springer (2008)

10. Künzle, V., Reichert, M.: Philharmonicflows: towards a framework for object-aware process management. Journal of Software Maintenance and Evolution: Research and Practice 23(4), 205-244 (2011)

11. Meroni, G., Baresi, L., Plebani, P.: Translating BPMN to E-GSM: specifications and rules. Tech. rep., Politecnico di Milano (2016), http://hdl.handle.net/ $11311 / 976678$

12. Meyer, A., Pufahl, L., Fahland, D., Weske, M.: Modeling and enacting complex data dependencies in business processes. In: Business Process Management Proceedings. pp. 171-186. Springer (2013)

13. Meyer, A., Weske, M.: Activity-centric and artifact-centric process model roundtrip. In: BPM Workshops. pp. 167-181. Springer (2013)

14. Pesic, M., Schonenberg, H., Van der Aalst, W.M.: Declare: Full support for looselystructured processes. In: Enterprise Distributed Object Computing Conference Proceedings. pp. 287-287. IEEE (2007)

15. Pichler, P., Weber, B., Zugal, S., Pinggera, J., Mendling, J., Reijers, H.A.: Imperative versus Declarative Process Modeling Languages: An Empirical Investigation. In: Business Process Management Workshops. pp. 383-394. Springer (2012)

16. Popova, V., Dumas, M.: From petri nets to guard-stage-milestone models. In: Business Process Management Workshops. pp. 340-351. Springer (2013)

17. Reichert, M., Weber, B.: Enabling flexibility in process-aware information systems: challenges, methods, technologies. Springer Science \& Business Media (2012)

18. Rozinat, A., van der Aalst, W.M.: Conformance checking of processes based on monitoring real behavior. Information Systems 33(1), 64-95 (2008)

19. Russell, N., Hofstede, A.H.M.T., Mulyar, N.: Workflow controlflow patterns: A revised view. Tech. Rep. BPM-06-22, BPM Center Report, BPMcenter.org (2006) 